\title{
Strong Carrier Lifetime Enhancement in GaAs Nanowires Coated with Semiconducting Polymer
}

\author{
Chaw Keong Yong, ${ }^{\dagger}$ Keian Noori, ${ }^{\ddagger}$ Qiang Gao, ${ }^{\S}$ Hannah J. Joyce, ${ }^{\dagger}$ H. Hoe Tan, ${ }^{\S}$ Chennupati Jagadish, ${ }^{\S}$ \\ Feliciano Giustino, ${ }^{\ddagger}$ Michael B. Johnston, ${ }^{\dagger}$ and Laura M. Herz ${ }^{*} \dagger$ \\ ${ }^{\dagger}$ Department of Physics, University of Oxford, Clarendon Laboratory, Parks Road, Oxford OX1 3PU, U.K. \\ ${ }^{\ddagger}$ Department of Materials, University of Oxford, Parks Road, Oxford OX1 3PK, U.K. \\ ${ }^{\S}$ Department of Electronic Materials Engineering, Research School of Physics and Engineering, Institute of Advanced Studies, The \\ Australian National University, Canberra ACT 0200, Australia
}

\section{Supporting Information}

ABSTRACT: The ultrafast charge carrier dynamics in GaAs/ conjugated polymer type II heterojunctions are investigated using time-resolved photoluminescence spectroscopy at $10 \mathrm{~K}$. By probing the photoluminescence at the band edge of GaAs, we observe strong carrier lifetime enhancement for nanowires blended with semiconducting polymers. The enhancement is found to depend crucially on the ionization potential of the polymers with respect to the Fermi energy level at the surface of the GaAs nanowires. We attribute these effects to electron doping by the polymer which reduces the unsaturated surface-

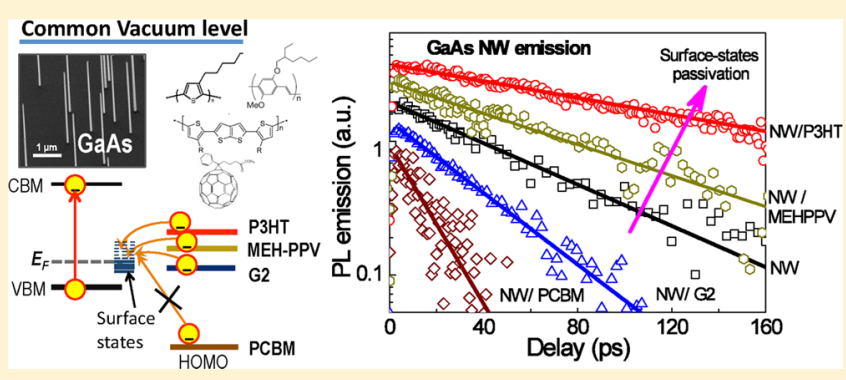
state density in GaAs. We find that when the surface of nanowires is terminated by native oxide, the electron injection across the interface is greatly reduced and such surface doping is absent. Our results suggest that surface engineering via $\pi$-conjugated polymers can substantially improve the carrier lifetime in nanowire hybrid heterojunctions with applications in photovoltaics and nanoscale photodetectors.

KEYWORDS: Nanowires, GaAs, organic semiconductors, ultrafast spectroscopy, hybrid photovoltaics, charge trapping

S olution processable, nanostructured hybrid materials consisting of organic and inorganic semiconducting components are highly attractive for the production of lowcost and high-efficiency solar cells. ${ }^{1-5}$ To obtain substantial photocurrents, interpenetrating networks based on electrondonor and -acceptor components are generally used to form a so-called bulk heterojunction $(\mathrm{BHJ})$ for which ultrafast exciton dissociation and charge generation occur primarily at the interface between the two material components. ${ }^{1,6-9}$ A variety of promising hybrid solar cells have been reported which use $\pi$ conjugated polymers, such as polythiophenes or polyphenylenevinylene derivatives, in conjunction with inorganic nanomaterials, such as gallium arsenide (GaAs) ${ }^{10}$ cadmium selenide $(\mathrm{CdSe}){ }^{1,6,11}$ indium phosphide $(\mathrm{InP}),{ }^{12}$ zinc oxide $(\mathrm{ZnO}),{ }^{2,13,14}$ or lead selenide $(\mathrm{PbSe}) .{ }^{15}$ Semiconductor nanowires (NWs) with high aspect ratio are particularly attractive for use in hybrid solar cells because they offer direct charge pathways to electrodes, large surface areas, high carrier mobility along the nanowires, and chemical and physical stability. $1,3,10,12,16,17$ However, the charge transport in such nanowires has been shown to be strongly affected by the presence of surface defect states ${ }^{18-20}$ that act as charge traps. A number of methods have therefore been devised to passivate such traps, including overgrowing the NWs surface with a layer of largebandgap semiconductor ${ }^{18,21}$ or treatment with sulfides. ${ }^{22}$
However, such overcoats may also form a barrier to charge generation at the interface of the nanowires when these are embedded in a $\pi$-conjugated polymer as part of a photovoltaic device structure. An excellent solution to this problem can be provided if conjugated polymers are to be found that provide both a $\mathrm{BHJ}$ interface with the nanowires but also effectively passivate the nanowire surface defects upon contact.

In this Letter, we report on how charge carrier lifetimes in GaAs nanowires are affected when the nanowires are embedded in a variety of $\pi$-conjugated polymers to form type II hybrid heterojunctions. We demonstrate that the PL lifetime of NWs embedded in the $\pi$-conjugated polymers can be enhanced significantly when the ionization potential (IP) of the polymer is smaller than the work function of the NWs and the native oxide layers on the surface of NWs are removed. X-ray photoemission spectroscopy reveals that the surface states of the uncoated GaAs NWs are electron-deficient in chemical character. Hence, we attribute the strong carrier lifetime enhancement to the filling of surface states in the nanowires by electron injection from the highest-occupied molecular orbital (HOMO) of the polymer into the energetically lower-lying

Received: September 12, 2012

Revised: November 14, 2012

Published: November 21, 2012 


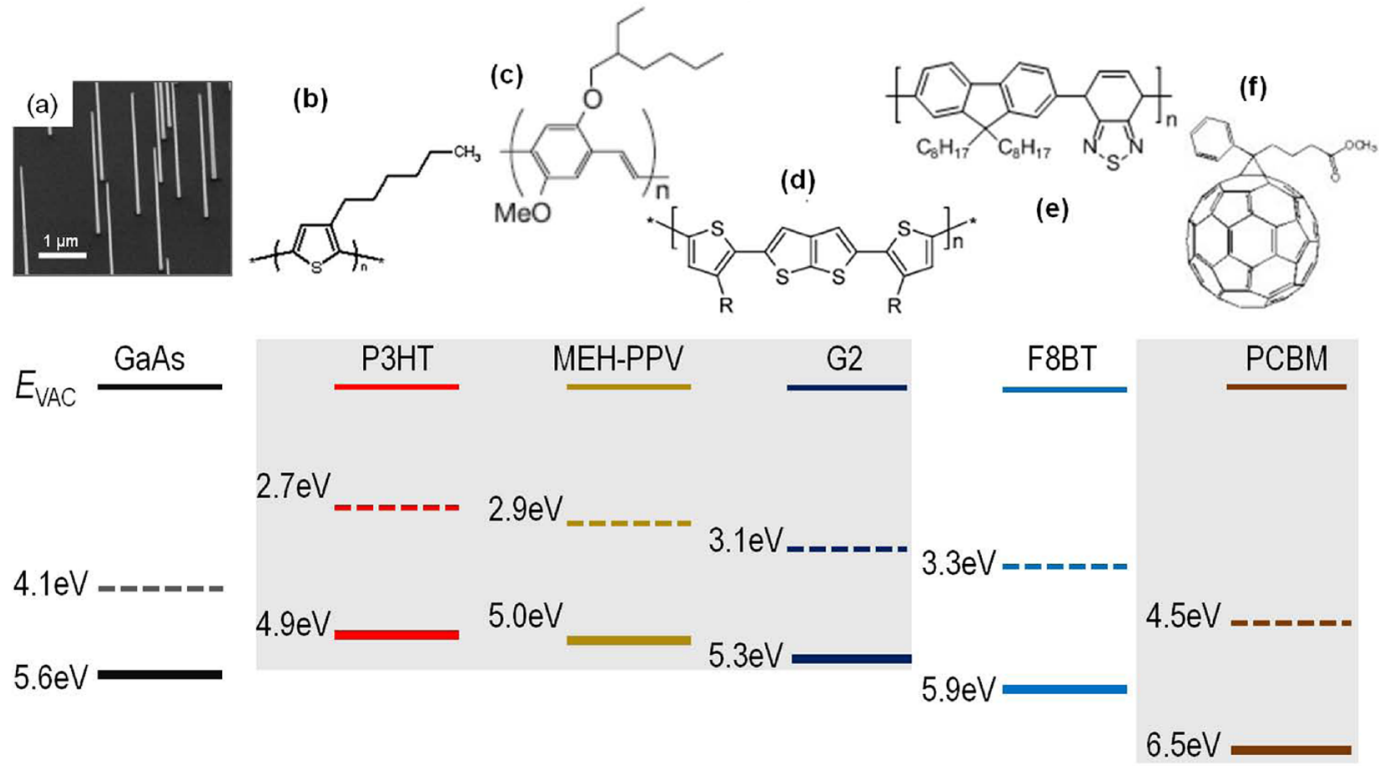

Figure 1. (a) Top: SEM image of GaAs nanowires as grown on an GaAs(111) substrate. Bottom: VBM and CBM energetic positions for bulk GaAs at $10 \mathrm{~K}$, taken from ref 23 . Note that due to their large diameter, these GaAs NWs experience negligible quantum confinement effects, and a bulk description is therefore appropriate here. ${ }^{21}$ Molecular structure (top) and HOMO/LUMO energy levels of (b) P3HT, (c) MEH-PPV, (d) G2, (e) F8BT, and (f) PCBM. The energetic levels of polymers are cited from refs 24 and 25 . The shaded areas indicate the formation of type II heterojunctions when GaAs nanowires are blended with P3HT, MEH-PPV, G2, or PCBM. For F8BT/GaAs nanowire blends a type I heterojunction is expected to form.

surface trap states of the nanowires until an equilibrium state is reached. To investigate such interfacial interactions, we performed density-functional theory calculations on a GaAs/ polythiophene heterojunction and find that the polymer becomes an electron-donating reagent to give a relatively thin ( $\approx 3 \AA$ ) "degenerate" electron-doping layer on the surface of GaAs. Our results demonstrate that charge lifetimes in GaAs nanowires can be significantly enhanced through overcoating with an organic semiconducting material of suitable IP that has the dual functions of acting as a surface passivating agent and being the secondary material component in the $\mathrm{BHJ}$ photovoltaic material.

The GaAs nanowires used in this study were fabricated on GaAs(111)B substrates using a metal-organic chemical vapor deposition (MOCVD) technique based on a previously described $^{18,26}$ two-temperature growth procedure which yields uniformly sized NWs with low bulk-defect density. Gold colloidal nanoparticles of $50 \mathrm{~nm}$ diameter were used as growth seeds, resulting in NWs of similar diameter, as shown in the SEM image displayed in Figure 1. The native oxide typically present on the surface of GaAs can be removed by established $\mathrm{HCl}$ solution etching methods. ${ }^{27}$ For our study, as-grown GaAs nanowires on $\mathrm{GaAs}(111)$ substrate were dipped into a $1 \mathrm{M}$ $\mathrm{HCl}$ solution for $30 \mathrm{~s}$ and rinsed with DI water for $10 \mathrm{~s}$ before being dried in a nitrogen gas stream. We examined the chemical nature of the surface states present in both as-grown and etched GaAs NWs by performing X-ray photoemission spectroscopy (XPS) measurements at the Daresbury National Centre for Electron Spectroscopy and Surface Analysis.

The GaAs NWs were subsequently embedded in $\mathrm{BHJ}$ thin films of various organic semiconducting materials, such as poly(3-hexylthiophene) (P3HT), poly(2-methoxy-5-(2-ethylhexyloxy)-1,4-phenylenevinylene) (MEH-PPV), poly(2,5-bis(3decylthiophen-2-yl)thieno(2,3-b)thiophene) (G2), [6,6]-phenyl-C61-butyric acid methyl ester (PCBM), and poly[(9,9-di- $n$ - octylfluorenyl-2,7-diyl)-alt-(benzo[2,1,3]thiadiazol-4,8-diyl)] (F8BT). Figure 1 displays the chemical structure of the organic semiconducting molecules used in this study. These materials have been widely used by others for a range of organic and hybrid organic-inorganic optoelectronic devices. ${ }^{5,6,10,25,28}$ All polymers were purchased from American Dye Source, except G2, which was synthesized by Merck Chemicals, as described in ref 25. The GaAs NWs/polymer blend films investigated in this study were prepared in an inert $\mathrm{N}_{2}$ atmosphere as follows. First, a $\mathrm{z}$-cut quartz substrate was spin-coated with a thin film of the organic semiconducting material, which had been dissolved in 1,2-dichlorobenzene (1,2-DCB) at a concentration of $0.2 \mathrm{mg} /$ $\mathrm{mL}$. To transfer the nanowires from their GaAs substrates onto these film-coated quartz substrates, we gently touched the two substrates together. This was then followed by drop-casting 30 $\mu \mathrm{L}$ of organic semiconductor solution onto the NWs distribution deposited on the initial films. Finally, the samples were dried on a hot-plate at $70{ }^{\circ} \mathrm{C}$ for $3 \mathrm{~h}$. The presence of the nanowires in the organic semiconductor compounds was clearly evident from the emerging photoluminescence spectra of these samples (see Supporting Information).

For time-integrated and time-resolved photoluminescence (PL) measurements, the sample was maintained at a temperature of $10 \mathrm{~K}$ in a liquid-helium flow cryostat. Time-resolved PL measurements were performed using a PL up-conversion setup that has already been described in detail elsewhere. ${ }^{29,30}$ The sample was excited at a photon energy of $1.68 \mathrm{eV}$ with the output from a mode-locked Ti:sapphire laser oscillator supplying $100 \mathrm{fs}$ pulses at $82 \mathrm{MHz}$ repetition rate. The PL was gated optically in a $\beta$-barium borate crystal using a split-off part of the laser output that was subjected to an adjustable time delay with respect to the excitation pulse. Time-resolved and time-integrated PL spectra were recorded with a liquidnitrogen-cooled CCD detector connected to a spectrometer and corrected for the spectral response of the apparatus. The 
spectral resolution of the time-resolved and time-integrated PL systems at the selected detection wavelengths was 32 and 4 $\mathrm{meV}$, respectively, and the former system had a time resolution of $200 \mathrm{fs}$.

Figure 2 shows the XPS spectra of GaAs NWs detected for the $\mathrm{Ga}_{\mathrm{GaAs}} 3 \mathrm{~d}$ and $\mathrm{As}_{\mathrm{GaAs}} 3 \mathrm{~d}$ core level regions. We have
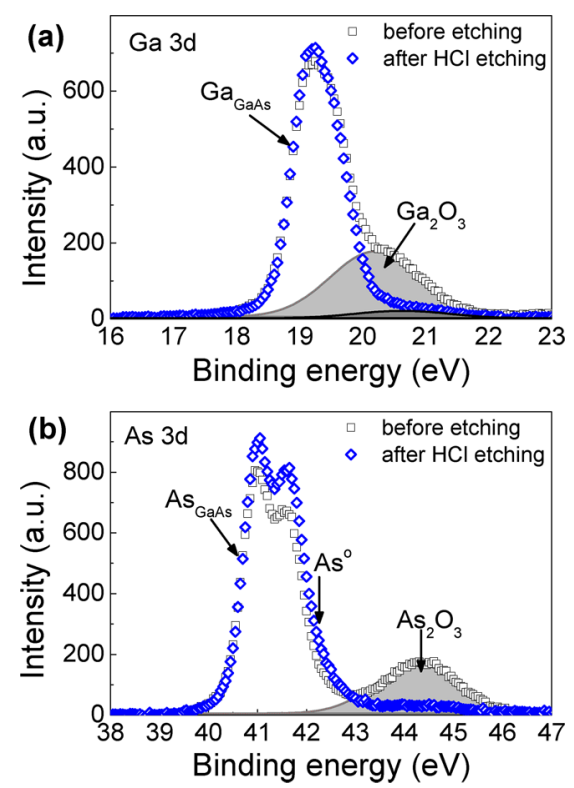

Figure 2. XPS spectra of GaAs nanowires before (as grown) and after etching with $\mathrm{HCl}$ solution, detected at the binding energy corresponding to (a) Ga 3d and (b) As 3d core energy levels. All XPS measurements were taken at a base pressure of $<10^{-9} \mathrm{mbar}$ and at room temperature.

previously observed in transmission electron microscopy (TEM) imaging that the surface of as-grown GaAs nanowires was terminated by an amorphous native oxide layer with thickness of $\approx 1 \mathrm{~nm}^{26}$ Since the XPS technique employed here probes a region of $\approx 2 \mathrm{~nm}$ below the NW surface, such native oxide layers ought to leave a clear fingerprint in the XPS spectra. Accordingly, we find a photoemission signal from asgrown GaAs NWs at a binding energy of $\approx 1.5 \mathrm{eV}$ above the $\mathrm{Ga}_{\mathrm{GaAs}} 3 \mathrm{~d}$ that can be assigned to the existence of $\mathrm{Ga}_{2} \mathrm{O}_{3}$ (see Figure 2). Similarly, the photoemission signal at a binding energy of $\approx 3.5 \mathrm{eV}$ above the $\mathrm{As}_{\mathrm{GaAs}} 3 \mathrm{~d}$ core level is attributed to the formation of $\mathrm{As}_{2} \mathrm{O}_{3} \cdot{ }^{31}$ In comparison, we find that for XPS spectra of $\mathrm{GaAs}$ nanowires treated with $\mathrm{HCl}$ solution both $\mathrm{Ga}_{2} \mathrm{O}_{3}$ and $\mathrm{As}_{2} \mathrm{O}_{3}$ signals are reduced tremendously with the small remaining signal probably caused by renewed oxidation during sample transfer through ambient atmosphere. These spectra hence demonstrate that the $\mathrm{HCl}$ solution treatment is highly effective in removing the native oxide layer present on the surface of as-grown nanowires. For clarification, we refer in the following to the untreated (as grown) and $\mathrm{HCl}$-solution treated GaAs NWs as $o$-GaAs NWs and $e$-GaAs NWs, respectively.

In further analysis, we extract the ratio of the integrated emission intensities of the $\mathrm{As}_{\mathrm{GaAs}} 3 \mathrm{~d}$ and $\mathrm{Ga}_{\mathrm{GaAs}} 3 \mathrm{~d}$ peaks and find that this ratio increases by $8 \%$ after the $\mathrm{HCl}$ solution treatment. In addition, the $\mathrm{As}_{\mathrm{GaAs}} 3 \mathrm{~d}$ signal is broadened at the high binding energy edge, which can be attributed to the presence of elemental $\mathrm{As}^{0}$ on the surface of nanowires. ${ }^{31} \mathrm{We}$ calculated the position of the Fermi level $E_{\mathrm{F}}$ from the $\mathrm{Ga}_{\mathrm{GaAs}}$ and $\mathrm{As}_{\mathrm{GaAs}} 3 \mathrm{~d}$ core levels (see Supporting Information) and find that $E_{\mathrm{F}}$ is located $\approx 0.5 \mathrm{eV}$ above the VBM of bulk GaAs. This suggests that the active surface sites of GaAs NWs are electron-deficient in chemical character. It should be noted that the peak positions of $\mathrm{Ga}_{\mathrm{GaAs}} 3 \mathrm{~d}$ and $\mathrm{As}_{\mathrm{GaAs}} 3 \mathrm{~d}$ do not change after the surface treatment, suggesting the Fermi level $\left(E_{\mathrm{F}}\right)$ pinning near the surface remains unchanged after the $\mathrm{HCl}$ solution treatment, as previously observed. ${ }^{32}$ Such $E_{\mathrm{F}}$ pinning below the midgap in GaAs has been attributed to the formation of donor states across the bandgap caused by the presence of excess As and an oxide layer. ${ }^{33,34}$ Since the nanowires under investigation here were grown under As-rich conditions, ${ }^{26}$ such surface-defect states are highly likely to be present near the nanowire surface. Therefore, the nanowires under investigation are characterized by the presence of chemical surface defects that render the NW surface p-type and present a significant trapping cross section to charge carriers.

We subsequently investigated the charge carrier dynamics in GaAs nanowires embedded in various organic semiconducting materials. Figure 1 displays a schematic of the relevant energy levels of bulk GaAs and the organic semiconductors for aligned vacuum levels $\left(E_{\mathrm{VAC}}\right)$. Type II heterojunctions are expected to form at the hybrid interface when GaAs NWs are blended with P3HT, MEH-PPV, G2, or PCBM. Specifically, hole injection from GaAs NWs into P3HT, MEH-PPV, and G2 should be favorable upon photoexcitation since the valence band maximum (VBM) and conduction band minimum (CBM) of $\mathrm{GaAs}$ are respectively deeper than the highest occupied molecular orbital (HOMO) and lowest unoccupied molecular orbital (LUMO) levels of these polymers. For GaAs NWs/ PCBM type II heterojunctions, on the other hand, electron injection from GaAs NWs into PCBM should follow photoexcitation, in accordance with the energetic offset at the interface shown in Figure 1. As a control sample, we also blended the GaAs NWs with F8BT for which an interfacial type I heterojunction ought to form at which photocarriers are confined in the GaAs NWs by the surrounding higher bandgap F8BT material. The slow drying and annealing processes employed when the hybrid films were produced generally induce arrangement of chains into more ordered domains. ${ }^{14,35-38}$ Films of P3HT produced under such conditions have been shown to exhibit large domains of lamellar structure and a high crystallinity, ${ }^{14,35,36}$ and those of PCBM also exhibit crystalline domains. ${ }^{39}$ For MEH-PPV, on the other hand, XRD profiles were shown to remain featureless even at high temperature, ${ }^{40}$ and the films thus can be considered to be amorphous.

To measure the charge carrier dynamics in GaAs nanowires, we conducted time-resolved PL measurements at a temperature of $10 \mathrm{~K}$. We excited the samples at a photon energy of $1.68 \mathrm{eV}$ and monitored the PL emission dynamics near the band edge of the GaAs NWs $\left(E_{\mathrm{g}}=1.52 \mathrm{eV}\right)$. The excitation density $(1.6 \times$ $10^{17} \mathrm{~cm}^{-3}$ ) was kept low in order to prevent PL quenching via carrier-carrier annihilation. ${ }^{21}$ While the exciting photon energy is far below the bandgap energy of the polymers used in this study, photoexcitations may still be generated in the polymer to some extent by weak two-photon absorption processes. However, we find that the PL signal originating from such two-photon transitions in the polymer is rather weak, as compared with the PL emerging from direct excitation of GaAs NWs, and is further quenched in the presence of GaAs NWs (see Supporting Information). PL spectra from NW/polymer blends are found to be dominated by emission from the GaAs 
nanowires at energies below $1.60 \mathrm{eV}$ (see Supporting Information), and the PL signal recorded at the photon energy of $1.52 \mathrm{eV}$ hence corresponds to band edge emission from the GaAs NWs.

Figure 3a shows the transient PL of untreated $o-G a A s$ NWs. From a single-exponential fit, we extract a PL lifetime of 46.5 ps
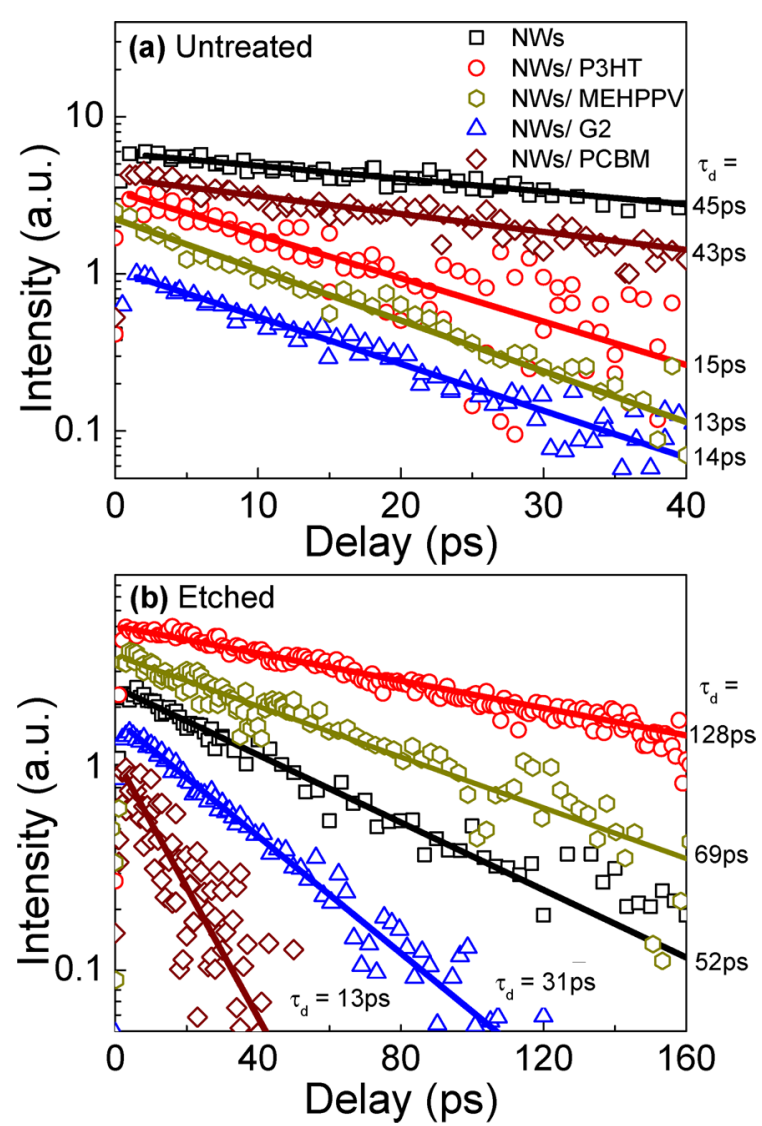

Figure 3. PL transients of (a) untreated (o-) and (b) etched (e-) GaAs NWs and their blends with a range of organic semiconductors, measured at the emission energy of $1.52 \mathrm{eV}$, close to the band edge of GaAs at $10 \mathrm{~K}$. The solid lines are the output of fitting these data with single-exponential decay functions of lifetime $\tau_{\mathrm{d}}$. for 0 -GaAs NWs. We have previously demonstrated that the short PL lifetime in bare (uncoated) GaAs nanowires is caused by a dominant nonradiative decay channel originating from charge trapping at surface-defect states. ${ }^{18,21}$ Following chemical etching which removes the surface native oxide, the PL lifetime of the $e$-GaAs NWs shows a marginal increase (52.1 ps), as displayed in Figure $3 \mathrm{~b}$. This suggests that for etched GaAs NWs, charge-carrier dynamics are still largely dominated by surface-defect states, in agreement with our observation from XPS measurement that the Fermi level near the surface remains unchanged upon etching. We further blended $e$-GaAs NWs with F8BT to form a hybrid type I heterojunction (Figure 1) for which charge transfer across the interface is expected to be absent and find that the PL decay trace remains essentially identical to those for bare e-GaAs NWs (see Supporting Information).

To investigate the carrier dynamics at a range of type II heterojunctions, we blended the GaAs nanowires with P3HT, MEH-PPV, G2, or PCBM. It has been generally observed that charge transfer across the interface of type II heterojunctions reduces the PL lifetime of the charge-donating material. ${ }^{7,8,41}$ Figure 3a shows the PL dynamics observed in $o$-GaAs NWs/ polymer heterojunction blends. Fast PL quenching is observed when $o$-GaAs NWs are blended with P3HT, MEH-PPV, and G2. This is attributable to hole transfer from GaAs NWs to the adjacent polymer layers, in accordance with their energetic level alignment shown in Figure 1. However, when these untreated nanowires are blended with PCBM, the PL recombination rate remains essentially unchanged from the PL observed in bare $o$ GaAs NWs. These data demonstrate that electron transfer from untreated GaAs NWs into the surrounding organic semiconductor is significantly slower than hole transfer.

Figure $3 \mathrm{~b}$ shows the contrasting PL dynamics for GaAs NWs that have been etched with $\mathrm{HCl}$ solution prior to being embedded in the same series of organic semiconductors. The PL dynamics appear more complex and are strikingly different from those observed for NWs covered in a native oxide layer. For the $e-G a A s$ NWs/PCBM heterojunction, nanowire PL quenching is now observed in accordance with efficient electron transfer from the NWs into PCBM. This result is to be expected given that the removal of the native oxide layer on

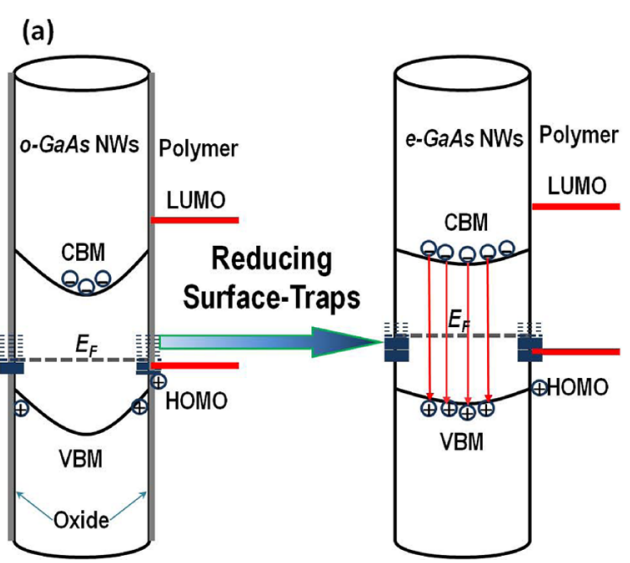

(b)

Common Vacuum level

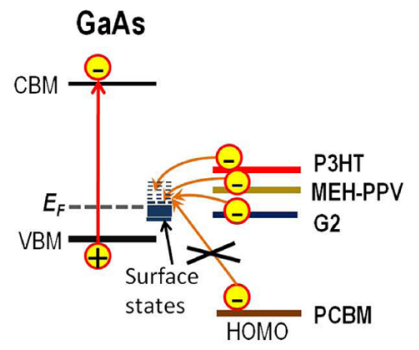

Figure 4. Schematic diagram illustrating the energy level alignment at the GaAs NW/organic semiconductor interface: (a) Schematic depicting bandbending effects across the nanowire cross section for $o$-GaAs NWs/polymer (left) and $e$-GaAs NWs/polymer (right) heterojunctions. For oxidized $o$ GaAs NWs the unpassivated surface leaves a p-doped surface that results in bending of the conduction (CB) and valence (VB) bands, while for $e$ GaAs NWs, charge injection from the polymers effectively passivates surface defects which leads to a flattening of the bands. (b) Common vacuum level picture indicating the passivation of NW surface states through electron transfer from the HOMO levels of P3HT, MEH-PPV, or G2. 
(a)

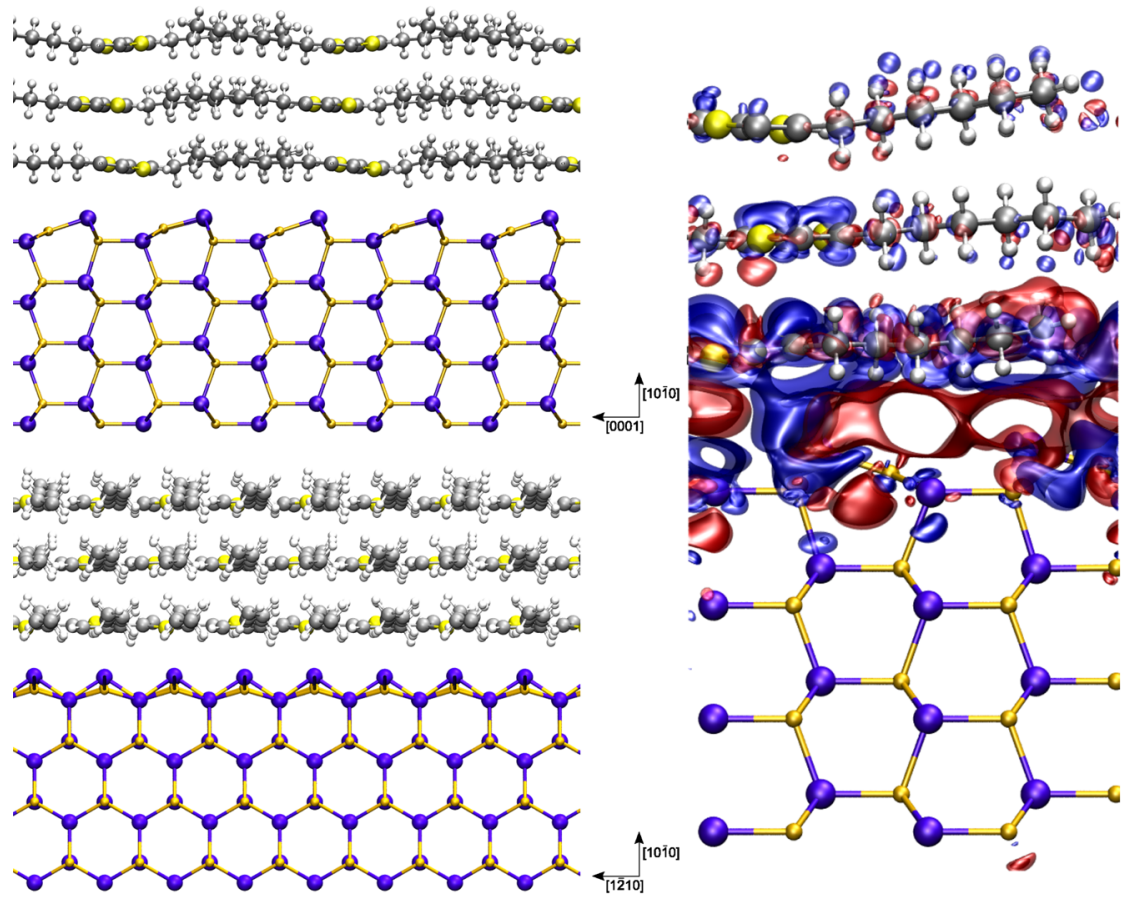

(c) charge density $\left(\times 10^{21}\right.$ electrons $\left./ \mathrm{cm}^{3}\right)$

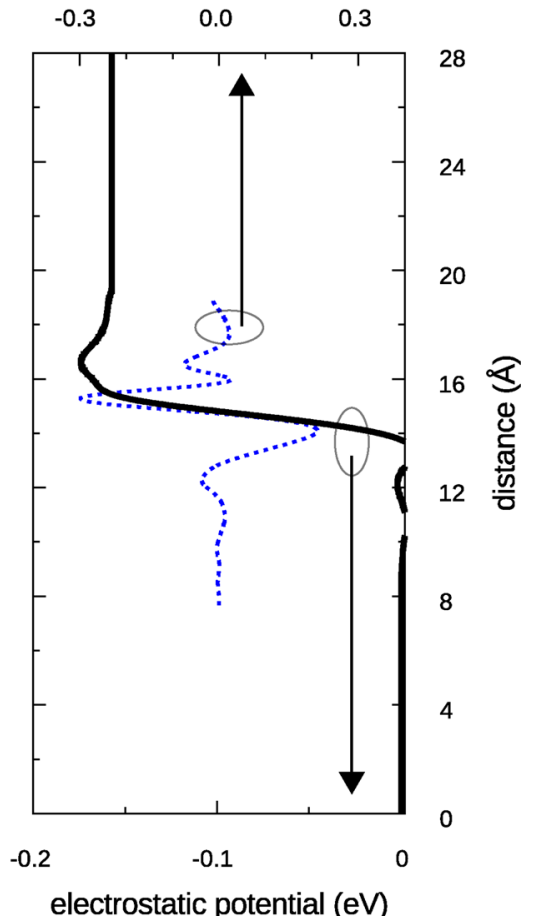

Figure 5. Density-functional theory modeling of the charge transfer at an idealized GaAs-P3HT interface. (a) Side views of the optimized and relaxed GaAs/P3HT interface with the P3HT backbone (top) and alkyl side chains (bottom) perpendicular to the page. The model comprises four layers of GaAs and three layers of P3HT (428 atoms). Atomic color code: Ga (violet), As (gold), O (red), S (yellow), C (gray), and H (white). (b) The isosurface of charge redistribution upon formation of the interface (isovalue: $\pm 6.75 \times 10^{-3}$ electrons/ $\AA^{3}$ for red/blue), calculated as $n_{\mathrm{GaAs} / \mathrm{P} 3 \mathrm{HT}}-$ $\left[n_{\mathrm{GaAs}}+n_{\mathrm{P} 3 \mathrm{HT}}\right], n(\mathbf{r})$ being the electron density at the position $\mathbf{r}$. (c) Planar average of the charge redistribution (dotted black line) and corresponding electrostatic potential profile across the interface (solid blue line), as obtained by integrating Poisson's equation. See Supporting Information for a detailed description of the computational methods used.

the surface of the nanowires ought to facilitate charge transfer across the NW/PCBM interface. Surprisingly, however, we find that the PL decay becomes slower following etching for $e$-GaAs NWs blended with G2, MEH-PPV, or P3HT. Figure 3b reveals that charge pair lifetimes in $e$-GaAs NWs blended with P3HT and MEH-PPV are even longer than those for bare (uncoated) $e$-GaAs NWs. Interestingly, the greatest PL lifetime enhancement is observed for the e-GaAs NWs blended with P3HT, which has the lowest IP of the polymers use in this study. These data suggest that following the removal of the native oxide layer, charge transfer across the $e$-GaAs NWs/polymer type II heterojunctions becomes strongly dependent on the alignment between the relevant energy levels in the polymer and the NWs.

The complex charge carrier dynamics we observe for the $\mathrm{NW}$ /organic semiconductor blends can be directly related to the modification of surface state density in the NWs by the organic semiconductor overcoating. The charge lifetime in the nanowires is altered by the surrounding organic semiconductor through two distinct mechanisms. First, the organic semiconductor offers a charge transfer pathway to photoexcited carriers generated in the nanowires, and second, it may passivate surface defect states in the GaAs NWs and hence eliminate charge trapping. The resulting changes in charge carrier lifetime in the nanowires can hence be understood quantitatively considering the surface band bending in the NWs, as illustrated in Figure 4a and explained in the following. Our XPS measurements reveal that the surface defect states in the uncoated nanowires are electron-deficient in chemical character and give $E_{\mathrm{F}}$ pinning below the midgap of GaAs. The resulting negative electric field at the surface bends the conduction and valence bands upward in energy, which spatially separates the electron and hole populations in the nanowires with holes accumulating near the surface and electrons near the center of the nanowires to maintain charge neutrality. ${ }^{34,42,43}$ Under low-intensity photoexcitation, holes can be driven to the surface of the nanowires by the built-in electric field while the electrons remain in the bulk of the nanowires, as illustrated in Figure 4a (left). Hence, the transfer of holes from the NWs into a surrounding material is expected to be much faster than the transfer of electrons which are inhibited by the surface potential barrier from reaching the surface. This scenario appears to be entirely consistent with the charge carrier dynamics observed in oxidized GaAs NW embedded in organic semiconductors (Figure $3 \mathrm{a}$ ). In the presence of $\approx 1 \mathrm{~nm}$ thick layer of native oxide, electron transfer from the $o$-GaAs NWs to PCBM is undetectably slow, while hole transfer to the MEH-PPV, G2, or P3HT polymers is fairly rapid. Consequently, the photoinduced charge transfer dynamics across the interface between the oxidized GaAs NWs and an organic semiconductor is governed by band-bending effects and cannot be portrayed within the simple picture of energetic offset across the interface under flat-band conditions.

However, once the surface of the GaAs NWs has been etched in order to remove the native oxide layer, significant changes are found to occur in the observed NW PL dynamics. Figure 3b 
demonstrates that removal of the NW surface oxide results in increased electron injection into PCBM and decreased hole injection into the polymers MEH-PPV, G2, or P3HT surrounding the NWs. The increased electron injection rate into PCBM is clearly expected given that an insulating oxide barrier has now been removed between the GaAs NW and the PCBM overcoat. However, following overcoating with conjugated polymers, contrary to expectation, a lengthening of charge carrier lifetimes is now observed in etched NWs in comparison to overcoated oxidized NWs. In particular, embedding the etched NWs in semiconducting polymers with the lowest ionization potentials (e.g., P3HT and MEH-PPV) is found to increase the lifetime of the PL emitted from the NWs with respect to that in uncoated NWs (Figure $3 \mathrm{~b}$ ). We have previously demonstrated that the PL dynamics from GaAs NWs is governed by strong charge trapping effects that result from the presence of a high density of surface defects in the GaAs NWs. ${ }^{18,21}$ Such charge trapping at surface states effectively competes with charge transfer from the nanowires into the organic semiconductor coating. Hence, the PL lifetime enhancement observed in GaAs NWs, as shown in Figure 3b, demonstrates that once the native oxide layers on the surface of NWs have been removed, the direct contact between the semiconducting polymer and the NWs must have reduced the surface states available for charge trapping. Such surface-defect passivation will reduce the extent of surface band bending as the Fermi energy near the surface is lifted toward the CBM of GaAs. ${ }^{34,42-44}$ This scenario is illustrated schematically in Figure $4 \mathrm{~b}$ : bringing an organic semiconductor with suitable IP into contact with the etched nanowire will lead to an initial electron injection into the NW until equilibrium is reached. This process leads to a reduction of charge trapping at surface states and a flattening of the bands, which in turn modifies the transfer of charges photogenerated inside the nanowires into the surrounding semiconductor since holes can now be more homogeneously distributed within the nanowires (see Figure $4 a$, right). In particular, data in Figure $3 \mathrm{~b}$ suggest that photoexcited holes in the etched nanowires experience slower transfer across the interface than in the absence of surface passivation. This is most likely so because holes are now to a lesser degree confined near the nanowire surface. Furthermore, the modification of the available density of states in the polymer following charge injection into NW surface states may also slow the transfer of photoexcited holes into the polymers. ${ }^{45}$

The emission lifetime enhancement we observe upon overcoating the etched GaAs NWs with conjugated polymers suggests that this method is highly effective at passivating surface defects. To understand the interfacial charge transfer between GaAs NWs and polymers that induces this passivation, we performed density-functional theory (DFT) calculations based on idealized GaAs/P3HT heterojunctions. It is currently beyond available computational power to incorporate into the DFT calculations the complexities of the realistic interfaces, which include surface states, nonstoichiometry, oxidation in the NWs, and a certain degree of packing disorder in the polymer. However, it will be insightful to assess the extent to which charge exchange occurs at the perfectly ordered and defect-free NW/P3HT interfaces simulated here.

Full details of the DFT computational methods are provided in the Supporting Information. Figure 5a shows the obtained optimized atomistic configuration of the $\mathrm{P} 3 \mathrm{HT}$ layers on the GaAs surface. The observed degree of $\mathrm{P} 3 \mathrm{HT}$ interdigitation is in line with both bulk values and calculated values reported for
$\mathrm{ZnO} / \mathrm{P} 3 \mathrm{HT}$ interface. ${ }^{46}$ Figure $5 \mathrm{~b}$ shows the isosurfaces of the charge-carrier redistributions upon formation of the GaAs/ $\mathrm{P} 3 \mathrm{HT}$ interface, with red (blue) regions indicating an increase (decrease) in electron density upon contact between the two materials. We find that interface formation is followed by an overall electron transfer from P3HT to GaAs that is spread across a surprisingly narrow region around the interface. Figure $5 \mathrm{c}$ shows the planar average of charge density redistribution across the interface, which are found to be largely localized within $\approx 3 \AA$ of that interface. Integration of these planar charge density profiles via Poisson's equation reveals that the charge redistribution results in an electrostatic potential offset of $\approx 0.2$ $\mathrm{eV}$ within the $3 \AA$ around the interface. Such strong charge localization at the interface is consistent with previous experimental findings for materials with low electron permittivity, for which charge carrier interactions at the interface are predominantly electrostatic. ${ }^{9}$ From the calculated charge redistribution we find that the surface of GaAs is degenerately doped at a charge carrier density of $\approx 1.2 \times 10^{13}$ $\mathrm{cm}^{-2}$, which corresponds to 0.07 holes per thiophene ring on P3HT for the simulated chain arrangement shown in Figure 5a. These results clearly suggest the feasibility of a $\delta$-electron doping layer forming on the surface of GaAs after it has been coated with P3HT.

Surface doping has previously been demonstrated in both organic and inorganic semiconductors through the use of surface functionalization with polar molecules, which allow controlled enhancement of the charge conductivity near the surface. $^{47-50}$ For GaAs surfaces, monolayer coverage with electron-donating reagents ${ }^{22}$ or polar molecules ${ }^{42}$ has been shown to effectively reduce the surface recombination velocity via charge transfer to the unsaturated surface states. ${ }^{43}$ In contrast, bulk doping of GaAs has been found to be fairly ineffective for passivating surface-defect states, in particular for high-quality n-doped GaAs, because of the formation of surface acceptor states by a self-compensation mechanism. ${ }^{51}$ Here we find that functional conjugated polymers, such as $\mathrm{P} 3 \mathrm{HT}$, can be highly effective electron-donating reagents for inorganic semiconductors as they appear to induce a $\delta$-electron doping layer on the surface of GaAs. Since the active surface states of the nanowires investigated here are electron-deficient in chemical character, electron doping will be essential to reduce the unsaturated surface-state density.

To quantify the extent to which surface-defect passivation occurs for the different organic semiconductors used as overcoats, we extracted the PL recombination rates $\left(k_{\mathrm{d}}\right)$ of the GaAs NW emission from the data shown in Figure 3. The results of single-exponential fitting procedures are shown in Figure 6 which displays the extracted decay rate constants as a function of energy level alignment parameter $\Delta_{\mathrm{IB}}$ and IP for bare-GaAs NWs and for GaAs NWs blended with P3HT, MEH-PPV, and G2. Here, $\Delta_{\mathrm{IB}}$ is the difference between the nominal valence band maximum $\mathrm{VBM}_{\mathrm{GaAs}}$ of bulk GaAs (5.6 $\mathrm{eV}$ ) and the HOMO level of the surrounding polymer when aligning their vacuum level.

For heterojunctions formed between oxidized $o$-GaAs NWs and polymers, the PL recombination rate $k_{\mathrm{d}}$ falls into the range of $0.065-0.071 \mathrm{ps}^{-1}$ and shows no dependence on $\Delta_{\mathrm{IB}}$. This result is thus in accordance with the $1 \mathrm{~nm}$ thick layer of native GaAs oxide preventing charge exchange between the two materials upon contact, leaving the surface traps on the GaAs NWs unpassivated. As a result, the PL recombination dynamics are governed by charge trapping at surface sites as previously 


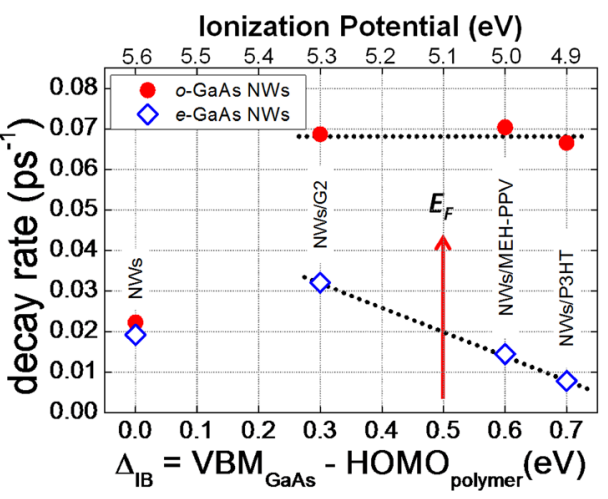

Figure 6. PL recombination rate $\left(k_{\mathrm{d}}\right)$ of $o$-GaAs NWs (full circles) and $e$-GaAs NWs (empty diamonds), uncoated or as part of a heterojunction with different polymers, as extracted by monoexponential fitting to the corresponding PL decay traces shown in Figure 3. $k_{\mathrm{d}}$ values are shown as a function of the ionization potential IP (top axis), where the IP for the polymer is used for NW/polymer heterojunctions and the IP of bulk GaAs is used for bare (uncoated) NWs. The bottom axis $\left(\Delta_{\mathrm{IB}}\right)$ shows the same scale as the top axis, but with the IP of bulk GaAs $(5.6 \mathrm{eV})$ subtracted in order to indicate the dependence of $\left(k_{\mathrm{d}}\right)$ on the IP offset between the two materials, as expected from common vacuum level considerations. The red arrow indicates the Fermi level $E_{\mathrm{F}}$ position on the surface of bare NWs (5.1 $\mathrm{eV}$ ) as inferred from XPS measurements. The dotted lines are guide to the eyes.

observed $^{21}$ and additional photoinduced injection of holes localized near the NW surface into the polymer (see Figure 4a, left) as discussed above. However, such photoinduced hole transfer appears to be independent of $\Delta_{\mathrm{IB}}$, which is expected given the large energetic offset at the interface (compared with the exciton binding energy of $4.5 \mathrm{meV}$ in GaAs) and the large variety of hole acceptor states available in the polymers. ${ }^{52}$

In marked contrast, the PL recombination rate $k_{\mathrm{d}}$ for $e$-GaAs NWs coated with conjugated polymer is significantly lower than that observed in $o$-GaAs NWs/polymer heterojunctions and decreases concomitantly with the increase of $\Delta_{\mathrm{IB}}$ across the heterojunctions. Strikingly, the emission lifetime for $e$-GaAs NWs increases (lower $k_{\mathrm{d}}$ ) when these nanowires are coated with either P3HT or MEH-PPV in accordance with effective passivation of NW surface trap states. We linearly extrapolate the data (see dashed line in Figure 6) and find that, for $\Delta_{\mathrm{IB}}>$ $0.5 \mathrm{eV}$ (corresponding to a polymer IP $<5.1 \mathrm{eV}$ ), the recombination rate of these type II heterojunction falls below the recombination rate observed in bare NWs. Interestingly, this value also corresponds to the Fermi level position $E_{\mathrm{F}}$ near the surface of the GaAs NWs, which our XPS measurements showed to lie $0.5 \mathrm{eV}$ above the $\mathrm{VBM}_{\mathrm{GaAs}}\left(\mathrm{VBM}_{\mathrm{GaAs}}=5.6 \mathrm{eV}\right.$ for bulk GaAs at $10 \mathrm{~K}^{23}$ ). This plot therefore confirms that the carrier lifetime enhancement observed in $e$-GaAs NWs/polymer heterojunctions is directly related to the filling of unsaturated nanowires surface states via electron doping from the HOMO of the $\pi$-conjugated polymer with the efficacy of the mechanism depending on the IP of the polymer, as shown in Figure 4b. In accordance with our DFT calculations, an interface dipole is formed concomitantly to lower the HOMO level of the polymer until the system attains equilibrium and further charge flow across the interface ceases. Hence, the semiconducting polymer with the lowest IP should give the highest surface-state filling, which is consistent with the data shown in Figure 6. Furthermore, the calculations suggest that this $\delta$-electron doping layer is largely localized in the close vicinity of the interface $(\approx 3 \AA)$. Accordingly, we find that the presence of $\approx 1$ $\mathrm{nm}$ native oxide on the surface of the nanowires is sufficient to prevent such passivation of defect states, as is the deposition of a $20 \mathrm{~nm}$ thick layer of AlGaAs on top of the GaAs nanowire core prior to polymer coating (see Supporting Information). Effective defect passivation of inorganic semiconductors with conjugated polymers thus requires both the correct choice of ionization potentials and sufficiently barrier-free interfaces to allow charge equilibration between the two components.

Our observations raise the question of how surface doping and sample morphology are expected to affect photovoltaic device performance. The strong localization of such doping effects near the interface, with most changes in the polymer occurring within the first monolayer at the interface, and the clear correlation with polymer IP suggest that energy level alignment is the dominant factor in determining the interfacial charge dynamics for this system. The extent of molecular ordering in the organic semiconductor, which varies strongly between the investigated materials, ${ }^{14,35-40}$ hence appears to play a less important role. However, molecular ordering is likely to have significant effects on the initial exciton diffusion and subsequent charge collection through the organic semiconductor, which is not investigated here but has a strong impact on the overall device performance of hybrid photovoltaic cells. ${ }^{14}$ As far as the overall light harvesting is concerned, our study shows that surface doping prolongs the lifetime of excited carriers in the nanowires, but it also appears to slow charge transfer across the hybrid interface, possibly due to a combination of reduced band bending and changes in the density of states in the charge-injecting organic. For use of these materials in photovoltaic devices, this means that the change in overall charge injection efficiency from nanowires into the organic may be small. However, such reduced charge transfer across the interface will also reduce recombination at a later stage. In addition, the reduction in charge trapping will allow better charge collection along the nanowires. Hence, the overall effect is likely to enhance the device efficiency, and further investigations of charge transport and recombination dynamics based on complete hybrid photovoltaic devices are needed to explore such effects.

In conclusion, we provide direct evidence for an effective passivation of surface defect states in GaAs nanowires through overcoating with conjugated polymers that establish a type II heterojunction. Upon removing the native oxide layer from the surface of the nanowires, we observe strong carrier lifetime enhancement for GaAs NWs overcoated with certain $\pi$ conjugated polymers. We attribute this effect to $\delta$-electron doping from the polymers to the surface of GaAs NWs, which reduces the unsaturated surface-state density. Quantitative analysis of the PL decay traces for all investigated heterojunctions reveals that the carrier-lifetime enhancement depends critically on the IP of the polymers: PL lifetime enhancement is only observed for nanowires blended with conjugated polymers whose IP is lower than the work function of the nanowires. Our observations suggest that electron injection from the HOMO of the polymer into the energetically lower lying surface states of the GaAs nanowires readily occurs during the formation of a hybrid type II heterojunction until the system comes to an equilibrium. While the picture that emerges superficially resembles that of a metal-semiconductor Schottky contact, ${ }^{34}$ a fundamental difference is the strong localization of passivating dopant carriers near the GaAs/ polymer surface: DFT calculations reveal that such doping is 
predominantly limited to the topmost layers near the surface ( $\approx 3 \AA$ ), and an interface dipole is formed concomitantly to lower the HOMO level of the $\pi$-conjugated polymer until the system attains equilibrium. Hence, when the surface of nanowires is covered by native oxide layer of thickness $\approx 1$ $\mathrm{nm}$, such surface doping of the nanowires becomes inhibited. Our study thus demonstrates that in nanowire/conjugated polymer type II hybrid blends the polymer can perform the dual function of hole transporter and nanowire surface passivating agent. The removal of native oxide on the surface of the nanowires and the use of polymers with sufficiently small IP are essential in order for effective trap passivation to occur. These findings are important for the design and implementation of new blend materials for hybrid photovoltaic devices and nanoscale photodetectors.

\section{ASSOCIATED CONTENT}

\section{S Supporting Information}

Details of nanowire growth. Description of XPS measurements and Fermi level determination. Time-resolved photoluminescence spectra of nanowires and blends with polymers. Photoluminescence transients of nanowires with various overcoats. Description of DFT calculations. This material is available free of charge via the Internet at http://pubs.acs.org.

\section{AUTHOR INFORMATION}

\section{Corresponding Author}

*E-mail: 1.herz@physics.ox.ac.uk.

\section{Notes}

The authors declare no competing financial interest.

\section{ACKNOWLEDGMENTS}

The authors acknowledge financial support from the Engineering and Physical Sciences Research Council (UK), the Australian Research Council, and the Australian National Fabrication Facility. The authors are grateful to Merck Chemicals for providing a quantity of polymer G2 and to Hung-Chun Lai for helpful discussions on XPS measurements. K.N. and F.G. are supported by the European Research Council under the EU FP7/ERC grant 239578. The calculations were performed in part at the Oxford Supercomputing Centre, and figures involving atomic structures were rendered using VMD. ${ }^{53}$

\section{REFERENCES}

(1) Huynh, W. U.; Dittmer, J. J.; Alivisatos, A. P. Science 2002, 295, $2425-2427$.

(2) Oosterhout, S. D.; Wienk, M. M.; Thiedmann, R.; Koster, L. J. A.; Gilot, J.; Loos, J.; Schmidt, V.; Janssen, R. A. J. Nat. Mater. 2009, 8, 818-824.

(3) Law, M.; Greene, L. E.; Johnson, J. C.; Saykally, R.; Yang, P. D. Nat. Mater. 2005, 4, 455-459.

(4) Dayal, S.; Kopidakis, N.; Olson, D. C.; Ginley, D. S.; Rumbles, G. Nano Lett. 2010, 10, 239-242.

(5) Szendrei, K.; et al. Adv. Mater. 2009, 21, 683-687.

(6) Ginger, D. S.; Greenham, N. C. Phys. Rev. B 1999, 59, 1062210629.

(7) Greenham, N. C.; Peng, X. G.; Alivisatos, A. P. Phys. Rev. B 1996, 54, 17628-17637.

(8) Wang, P.; Abrusci, A.; Wong, H. M. P.; Svensson, M.; Andersson, M. R.; Greenham, N. C. Nano Lett. 2006, 6, 1789-1793.

(9) Nĕmec, H.; Rochford, J.; Taratula, O.; Galoppini, E.; Kužel, P.; Polìka, T.; Yartsev, A.; Sundström, V. Phys. Rev. Lett. 2010, 104, 197401.
(10) Ren, S. Q.; Zhao, N.; Crawford, S. C.; Tambe, M.; Bulovic, V.; Gradečak, S. Nano Lett. 2011, 11, 408-413.

(11) Dayal, S.; Reese, M. O.; Ferguson, A. J.; Ginley, D. S.; Rumbles, G.; Kopidakis, N. Adv. Funct. Mater. 2010, 20, 2629-2635.

(12) Novotny, C. J.; Yu, E. T.; Yu, P. K. L. Nano Lett. 2008, 8, 775779 .

(13) Beek, W. J. E.; Wienk, M. M.; Janssen, R. A. J. Adv. Funct. Mater. 2006, 16, 1112-1116.

(14) Briseno, A. L.; Holcombe, T. W.; Boukai, A. I.; Garnett, E. C.; Shelton, S. W.; Frechet, J. J. M.; Yang, P. D. Nano Lett. 2010, 10, 334340.

(15) Cui, D. H.; Xu, J.; Zhu, T.; Paradee, G.; Ashok, S.; Gerhold, M. Appl. Phys. Lett. 2006, 88, 183111.

(16) Schierhorn, M.; Boettcher, S. W.; Peet, J. H.; Matioli, E.; Bazan, G. C.; Stucky, G. D.; Moskovits, M. ACS Nano 2010, 4, 6132-6136.

(17) Lin, Y. Y.; Chu, T. H.; Li, S. S.; Chuang, C. H.; Chang, C. H.; Su, W. F.; Chang, C. P.; Chu, M. W.; Chen, C.-W. J. Am. Chem. Soc. 2009, 131, 3644-3649.

(18) Parkinson, P.; Joyce, H. J.; Gao, Q.; Tan, H. H.; Zhang, X.; Zou, J.; Jagadish, C.; Herz, L. M.; Johnston, M. B. Nano Lett. 2009, 9, 3349-3353.

(19) Haight, R.; Sekaric, L.; Afzali, A.; Newns, D. Nano Lett. 2009, 9, 3165-3170.

(20) Demichel, O.; Calvo, V.; Besson, A.; Noe, P.; Salem, B.; Pauc, N.; Oehler, F.; Gentile, P.; Magnea, N. Nano Lett. 2010, 10, 23232329.

(21) Yong, C. K.; Joyce, H. J.; Lloyd-Hughes, J.; Gao, Q.; Tan, H. H.; Jagadish, C.; Johnston, M. B.; Herz, L. M. Small 2012, 8, 1725.

(22) Lunt, S. R.; Ryba, G. N.; Santangelo, P. G.; Lewis, N. S. J. Appl. Phys. 1991, 70, 7449-7465.

(23) Blakemore, J. S. J. Appl. Phys. 1982, 53, R123.

(24) Chua, L. L.; Zaumseil, J.; Chang, J. F.; Ou, E. C. W.; Ho, P. K. H.; Sirringhaus, H.; Friend, R. H. Nature 2005, 434, 194-199.

(25) Heeney, M.; Bailey, C.; Genevičius, K.; Shkunov, M.; Sparrowe, D.; Tierney, S.; McCulloch, I. J. Am. Chem. Soc. 2005, 127, 10781079.

(26) Joyce, H. J.; Gao, Q.; Tan, H. H.; Jagadish, C.; Kim, Y.; Zhang, X.; Guo, Y. N.; Zou, J. Nano Lett. 2007, 7, 921-926.

(27) Traub, M. C.; Biteen, J. S.; Brunschwig, B. S.; Lewis, N. S. J. Am. Chem. Soc. 2008, 130, 955-964.

(28) Zaumseil, J.; Donley, C. L.; Kim, J. S.; Friend, R. H.; Sirringhaus, H. Adv. Mater. 2006, 18, 2708-2712.

(29) Schmid, S. A.; Abbel, R.; Schenning, A. P. H.; Meijer, E. W.; Sijbesma, R. P.; Herz, L. M. J. Am. Chem. Soc. 2009, 131, 17696.

(30) Chang, M. H.; Hoeben, F. J. M.; Jonkheijm, P.; Schenning, A. P. H. J.; Meijer, E. W.; Silva, C.; Herz, L. M. Chem. Phys. Lett. 2006, 418, 196.

(31) Surdu-bob, C. C.; Saied, S. O.; Sullivan, J. L. Appl. Surf. Sci. 2001, 183, 126-136.

(32) Szuber, J.; Bergignat, E.; Hollinger, G.; Polakowska, A.; Koscielniak, P. Vacuum 2002, 67, 53-58.

(33) Lelay, G.; Mao, D.; Kahn, A.; Hwu, Y.; Margaritondo, G. Phys. Rev. B 1991, 43, 14301-14304.

(34) Spicer, W. E.; Lilientalweber, Z.; Weber, E.; Newman, N.; Kendelewicz, T.; Cao, R.; McCants, C.; Mahowald, P.; Miyano, K.; Lindau, I. J. Vac. Sci. Technol., B 1988, 6, 1245-1251.

(35) Rodd, C. M.; Agarwal, R. Nano Lett. 2011, 11, 3460-3467.

(36) Ho, P. K. H.; Chua, L. L.; Dipankar, M.; Gao, X. Y.; Qi, D. C.; Wee, A. T. S.; Chang, J. F.; Friend, R. H. Adv. Mater. 2007, 19, 215221.

(37) Bearden, S. D.; Cannon, J. P.; Gold, S. A. Macromolecules 2011, 44, 2200-2205.

(38) Li, G.; Yao, Y.; Yang, H.; Shrotriya, V.; Yang, G.; Yang, Y. Adv. Funct. Mater. 2007, 17, 1636-1644.

(39) Nam, S.; Jang, J.; Cha, H.; Hwang, J.; An, T. K.; Park, S.; Park, C. E. J. Mater. Chem. 2012, 22, 5543-5549.

(40) Chen, S. H.; Su, A. C.; Huang, Y. F.; Su, C. H.; Peng, G. Y.; Chen, S. A. Macromolecules 2002, 35, 4229-4232. 
(41) Stranks, S. D.; Weisspfennig, C.; Parkinson, P.; Johnston, M. B.; Herz, L. M.; Nicholas, R. J. Nano Lett. 2011, 11, 66-72.

(42) Cohen, R.; Kronik, L.; Shanzer, A.; Cahen, D.; Liu, A.; Rosenwaks, Y.; Lorenz, J. K.; Ellis, A. B. J. Am. Chem. Soc. 1999, 121, 10545-10553.

(43) Seker, F.; Meeker, K.; Kuech, T. F.; Ellis, A. B. Chem. Rev. 2000, 100, 2505-2536.

(44) Hasegawa, H.; Ishii, H.; Sawada, T.; Saitoh, T.; Konishi, S.; Liu, Y. A.; Ohno, H. J. Vac. Sci. Technol., B 1988, 6, 1184-1192.

(45) Vaynzof, Y.; Kabra, D.; Zhao, L. H.; Ho, P. K. H.; Wee, A. T. S.; Friend, R. H. Appl. Phys. Lett. 2010, 97, 033309-033311.

(46) Noori, K.; Giustino, F. Adv. Funct. Mater. 2012, DOI: 10.1002/ adfm.201201478.

(47) Kobayashi, S.; Nishikawa, T.; Takenobu, T.; Mori, S.; Shimoda, T.; Mitani, T.; Shimotani, H.; Yoshimoto, N.; Ogawa, S.; Iwasa, Y. Nat. Mater. 2004, 3, 317-322.

(48) He, T.; Corley, D. A.; Lu, M.; He, J. L.; Nackashi, D. P.; Franzon, P. D.; Tour, J. M. J. Am. Chem. Soc. 2009, 131, 10023-10030. (49) Shalom, M.; Ruhle, S.; Hod, I.; Yahav, S.; Zaban, A. J. Am. Chem. Soc. 2009, 131, 9876-9877.

(50) Calhoun, M. F.; Sanchez, J.; Olaya, D.; Gershenson, M. E.; Podzorov, V. Nat. Mater. 2008, 7, 84-89.

(51) Pashley, M. D.; Haberern, K. W.; Feenstra, R. M.; Kirchner, P. D. Phys. Rev. B 1993, 48, 4612-4615.

(52) Tvrdy, K.; Frantsuzov, P. A.; Kamat, P. V. Proc. Natl. Acad. Sci. U. S. A. 2011, 108, 29-34.

(53) Humphrey, W.; Dalke, A.; Schulten, K. J. Mol. Graphics 1996, $14,33-38$. 